\title{
MONOCULAR VS BINOCULAR 3D REAL-TIME BALL TRACKING FROM 2D ELLIPSES
}

\author{
Nicola Greggio ${ }^{\oslash} \ddagger$, José Gaspar ${ }^{\ddagger}$, Alexandre Bernardino ${ }^{\ddagger}$, José Santos-Victor ${ }^{\ddagger}$ \\ ${ }^{-}$ARTS Lab - Scuola Superiore S.Anna, Polo S.Anna Valdera, Viale R. Piaggio, 34 - 56025 Pontedera, Italy \\ $\ddagger$ Instituto de Sistemas e Robótica, Instituto Superior Técnico, 1049-001 Lisboa, Portugal \\ (ngreggio, alex,jag, jasv)@isr.ist.utl.pt
}

Keywords: Machine Vision, Pattern Recognition, Least-Square Fitting, Algebraic Distance.

\begin{abstract}
Real-Time tracking of elliptical objects, e.g. a ball, is a well studied field. However, the question between a monocular and binocular approach for 3D objects localization is still an open issue. In this work we implemented a real-time algorithm for 3D ball localization and tracking from 2D image ellipse fitting with calibrated cameras. We will exploit both approaches, together with their own characteristics. Our algorithm features the following key features: (1) a real-time video segmentation by means of a Gaussian mixture descriptor; (2) a closed-form ellipse fitting algorithm; and (3) a novel 3D reconstruction algorithm for spheres from the 2D ellipse parameters. We test the algorithm's performance in several conditions, by performing experiments in virtual scenarios with ground truth. Finally, we show the results of monocular and binocular reconstructions and evaluate the influence of having prior knowledge of the ball's dimension and the sensitivity of binocular reconstruction to mechanical calibration errors.
\end{abstract}

\section{INTRODUCTION}

3D object localization and tracking are leading subjects robotics and computer vision. The current ever and ever improvements in hardware capabilities (framegrabbers, motor controllers) require ever and ever more sophisticated techniques and algorithms to coope with. This is because the correct object/target identification in terms of 3D position, dimension, velocity, and trajectory is of primary importance to many applications. Vehicle guidance (Stentz, 2001) (Shi et al., 2007), rescue (Carpin et al., 2006) (S. Balakirsky et al., 2007), soccer robots (Menegatti et al., 2008) (Assfalg et al., 2003), or surveillance systems (Ottlik and Nagel, 2008) (Davis et al., 2006) are only few examples of fields that exploit this concept. In general unstructured and dynamic environments, tracking techniques must be robust, to cope with world uncertainty and sensor noise, and computationally efficient to provide fast reaction times to unexpected events.

Robustness is closely linked to the environment the application has to perform into. For instance, in industrial applications (i.e. in structured scenarios) a non-robust algorithm can perform well because of the absence of disturbances. However, in non-constrained scenarios this is no longer true: changes in color, shape and speed of the object can significantly perturb object identification and tracking. For instance in outdoor vision and robotics applications, like video surveillance and rescue robots, there is the need of a substantial adaptability toward the changing lighting and color conditions. The other fundamental issue required by a vision identification algorithm is its computational efficiency. For example in video surveillance, a precise but very slow algorithm leads to high (and sometimes unacceptable) latency time between the image acquisition and its feature extraction. Also in vision-based control applications, like robotics or vehicle guidance, low latencies are essential for the stability and performance properties of the control loops.

\subsection{Related Work}

Stereovision tracking requires the usage of two or more calibrated cameras that capture the same image at the same time from different known positions 
and orientation. An addressing example are the two cameras in a humanoid robot. Each image (left and right is processed separately in order to isolate the target). Then, thanks to the known robot's direct kinematics and encoders information the target position is retrieved by means of geometric triangulation (Forsyth and Ponce, 2002). In 2004, Kwolek developed a method for tracking human heads with a mobile stereovision camera (Kwolek, 2004). He characterized faces by first performing a color filtering, and then by modeling the head in the 2D image domain as an ellipse. Therefore, they formulated the tracking problem as a probabilistic one in which a particle filter is used to approximate the probability distribution by a weighted color cue, shape information, and stereovision sample collection. In 2011, Greggio et Al. implemented a $3 \mathrm{D}$ tracker for the iCub platform featuring an ellipse pattern recognition algorithm for the target description (Greggio et al., 2011). In their work, the authors performed a simple color based image filtering in order to evidence a green ball. Then, the $2 \mathrm{D}$ information obtained with both the iCub's cameras has been processed by the ellipse detector, and the results triangulated in order to reconstruct the ball's position.

On the other hand, monocular tracking features the usage of a single camera. Therefore all the triangulation information are lost, making this category more challenging than the previous one. Many exertions go toward the human beings tracking. Face tracking is one of the most important application for both video surveillance or simple people detection. In their work, Gokturk et Al. proposed tho apply the Principal Component Analysis to learn all the possible facial deformations (Gokturk et al., 2001). Then, they tracked pose and deformation of the face from an image sequence. Fossati et $\mathrm{Al}$. used a motion model to infer 3-D poses between consecutive detections for identifying key postures for recognizing people seen from arbitrary viewpoints by a single and potentially moving camera (Fossati et al., 2007). In 2010 Andriluka et $A l$. proposed a people spatial pose estimation technique based on three stages: Initial estimate of the 2D articulation from single frames, data association across frames, and recovering 3D pose (Andriluka et al., 2010).

\subsection{Main contributions}

In this work we present a real-time algorithm that robustly segments color blobs modeled by a finite Gaussian mixture model, following the approach proposed in (Greggio et al., 2010a) and in (Greggio et al., 2010b). This is an essential pre-processing step that allows the identification of connected components in the image where the remaining phases will be applied. Then, we present a novel algorithm to compute the 3D position of a world sphere corresponding to the projected ellipse in the camera. We performed several experiments in the simulator of the iCub robot (Tikhanoff et al., 2008) in order to verify our approach's performance in a realistic context. We compare, the reconstructions obtained with monocular and binocular approaches with ground truth data. Besides, we consider the existence or absence of $a$ priory knowledge about the ball's dimensions.

\subsection{Paper Organization}

This paper is organized as follows. In sec. 2 we will describe our mapping from $2 \mathrm{D}$ to $3 \mathrm{D}$ coordinates. Then, in sec. 3 we will describe our experimental setup and we will discuss our results. Finally, we will conclude and point out directions for future work in sec. 4.

\section{3D RECONSTRUCTION FROM ELLIPSES}

In this section we describe how to reconstruct the 3D location of the ball given monocular and binocular images of the ball silhouette and, in the monocular case, the knowledge of the ball radius.

\subsection{Projection Equation}

Describing the ball as a quadric surface, solution of the quadratic equation:

$$
X^{T} Q X=0,
$$

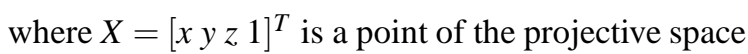
belonging to the ball surface, and its imaged silhouette (ellipse) as a conic:

$$
m^{T} \mathrm{Cm}=0
$$

where $m=\left[\begin{array}{lll}u & v & 1\end{array}\right]^{T}$ is a point of the projective plane belonging to the imaged silhouette, then the projection of the ball $Q$ to the ellipse $C^{1}$ is simply (Cross and Zisserman, 1998):

$$
C^{*} \sim P Q^{*} P^{T}
$$

where $P$ is a $3 \times 4$ projection matrix, $C^{*}=\operatorname{adj}(C)$ and $Q^{*}=\operatorname{adj}(Q)$ are the dual conic and the dual quadratic of $C$ and $Q$ respectively, and $\sim$ denotes equality up to a scale factor.

\footnotetext{
${ }^{1}$ In order to simplify the notation, we refer the parameter matrices $Q$ and $C$ interchangeably with the associated quadratic and conic equations.
} 


\subsection{Monocular Reconstruction}

Given the particular case of a spherical ball, the quadratic $Q$ has a simplified form. Considering a ball with radius $R$ centered at the origin, one has $Q_{0}=\operatorname{diag}\left(1,1,1,-R^{2}\right)$. Translation of the ball to a generic 3D location $t=\left[\begin{array}{ll}t_{x} & t_{y} \\ t_{z}\end{array}\right]^{T}$ is obtained by applying the homogeneous coordinates transformation $T$ to $Q_{0}$ as $Q=T Q_{0} T^{T}$, with $T=\left[I_{3}-t ; 0_{3}^{T} 1\right]$ where $I_{3}$ is a $3 \times 3$ identity matrix and $0_{3}$ is a vector of zeros. The translated quadratic has therefore the form:

$$
Q=\left[\begin{array}{cc}
I_{3} & -t \\
-t^{T} & t^{T} t-R^{2}
\end{array}\right]
$$

Noting that $C$ and $Q$ are symmetric matrices, then the adjoint matrices coincide with their inverses. Hence, the projection equation Eq.3 can be written as $C^{-1} \sim P Q^{-1} P^{T}$, with:

$$
Q^{-1}=\left[\begin{array}{cc}
I_{3}-t t^{T} / R^{2} & -t / R^{2} \\
-t^{T} / R^{2} & -1 / R^{2}
\end{array}\right]
$$

Considering, without loss of generality, that a camera has its coordinate frame coincident with the world frame $P=K\left[\begin{array}{ll}I_{3} & 0_{3}\end{array}\right]$, where $K$ denotes the intrinsics parameters matrix, one obtains $C^{-1} \sim K\left(I_{3}-\right.$ $\left.t t^{T} / R^{2}\right) K^{T}$. Given that $K$ is an invertible matrix one obtains the normalized projection equation:

$$
I_{3}-t t^{T} / R^{2} \sim K^{-1} C^{-1} K^{-T}
$$

Computing the characteristic equation of the LHS of Eq.6, $\operatorname{det}\left(I_{3}-t t^{T} / R^{2}-\lambda I_{3}\right)=0$ one finds that the LHS matrix has the eigenvalues $\lambda_{1,2,3}=\{1,1,1-$ $\left.\|t / R\|^{2}\right\}^{2}$. The two unitary eigenvalues of the LHS imply that the RHS has also two equal eigenvalues, but usually not-unitary given that the equality is only up to a scale factor. However, this observation allows finding a scale for the RHS that makes it equal to the LHS. The scaling has just to impose that the equal eigenvalues of the RHS are scaled to become unitary, which can be done by sorting the three eigenvalues and selecting the middle one.

More specifically, defining $H$ as the normalized conic of the RHS of Eq.6, i.e. $H=K^{-1} C^{-1} K^{-T}$, one removes the scale ambiguity by computing the median of the eigenvalues:

$$
I_{3}-t t^{T} / R^{2}=H / \operatorname{median}(\operatorname{eig}(H))
$$

where $\operatorname{eig}(H)$ denotes a function returning the set of three eigenvalues of $H$.

\footnotetext{
${ }^{2}$ Despite appearing that $\lambda_{3}=1-\|t / R\|^{2}$ can be zero, hence posing questions about the existence of $Q$, this is not a case of practical importance since $\lambda_{3}=0$ implies $\|t\|=R$, meaning that the ball would touch the camera projection center which is not possible in practice.
}

Removed the scale ambiguity, Eq.7 allows solving easily for the direction of the ball location $v=t / R$. Denoting $\mathrm{H}_{2}=\mathrm{I}_{3}-\mathrm{H} /$ median $(\operatorname{eig}(H))$, one obtains $v v^{T}=H_{2}$ and the direction of the ball location in the camera frame is:

$$
v=\left[\begin{array}{l}
s_{1} \sqrt{H_{2(1,1)}} \\
s_{2} \sqrt{H_{2(2,2)}} \\
s_{3} \sqrt{H_{2(3,3)}}
\end{array}\right]
$$

where $s_{1}, s_{2}, s_{3}$ denote the signs of the components of $v$. Assuming that the ball is in front of the camera, and the optical axis of the camera is the $z$ axis, pointing forward, then $s_{3}=+1$ and one can compute $s_{1}=\operatorname{sign}\left(H_{2}(1,3)\right), s_{2}=\operatorname{sign}\left(H_{2}(2,3)\right)$ where $\operatorname{sign}($. is the sign function returning $+1,-1,0$ for positive, negative or null arguments respectively. Finally, given the ball radius, $R$ computing the ball location in the camera frame coordinate, $t$ is just a scaling of the computed direction:

$$
t=R v .
$$

Computing the ball location in world coordinates, ${ }^{w} t$, given a generic projection matrix, $P=K\left[{ }^{c} R_{w}{ }^{c} t_{w}\right]$, proceeds as before, firstly using just the intrinsic parameters $K$, and than correcting the computed pose to the world coordinates frame:

$$
{ }^{w} t={ }^{c} R_{w} t+{ }^{c} t_{w}
$$

Note that even if $P$ is not given in a factorized form, the intrinsic $K$ and extrinsic parameters ${ }^{c} R_{w},{ }^{c} t_{w}$ can be extracted from $P$ using QR factorization as described in (Hartley and Zisserman, 2000).

\subsection{Binocular Reconstruction}

Considering a binocular vision system, Eq.3 is replicated for the two cameras:

$$
\left\{\begin{array}{l}
C_{1}^{-1} \sim P_{1} Q^{-1} P_{1}^{T} \\
C_{2}^{-1} \sim P_{2} Q^{-1} P_{2}^{T}
\end{array}\right.
$$

where $P_{1}$ and $P_{2}$ denote the two projection matrices, possibly having different intrinsic matrices, $K_{1}, K_{2}$, rotation matrices, ${ }^{c 1} R_{w},{ }^{c 2} R_{w}$, and camera centers, ${ }^{c 1} t_{w},{ }^{c 2} t_{w}$.

In the case of knowing the radius of the ball, $R$ then the monocular reconstruction methodology can be applied twice, resulting in two estimates of the ball location, ${ }^{w} t_{1},{ }^{w} t_{2}$, from which one can obtain a final estimate as the mean of the two estimates, ${ }^{w} t=\left({ }^{w} t_{1}+\right.$ $\left.{ }^{w} t_{2}\right) / 2$

In case of not knowing the radius of the ball, a binocular (stereo) setup still allows obtaining depth estimates by triangulation, provided that the setup has a not-null baseline, i.e. different camera centers, ${ }^{w} t_{c 1} \neq{ }^{w} t_{c 2}$ where ${ }^{w} t_{c i}=-{ }^{c i} R_{w}^{-1 c i} t_{w}$ and ${ }^{w} t_{c i}=$ 
$-P_{i(1: 3,1: 3)}^{-1} P_{i(1: 3,4)}$ for $i=1,2$. Using Eq.8, one obtains two directions to the ball location, $v_{1}, v_{2}$, which can be converted to a common (world) coordinate system, ${ }^{w} v_{i}={ }^{c i} R_{w}^{-1} v_{i}, i=1,2$. The ball location can therefore be obtained by scaling these directions, starting at the camera centers, ${ }^{w} t_{c i}$, and finding the closest to an intersection point in a least squares sense:

$$
\left(\alpha^{*}, \beta^{*}\right)=\arg _{\alpha, \beta} \min \left\|\alpha^{w} v_{1}+{ }^{w} t_{c 1}-\left(\beta^{w} v_{2}+{ }^{w} t_{c 2}\right)\right\|^{2} .
$$

Collecting $\alpha, \beta$ into a vector, the cost function of Eq.11 can be rewritten as $\left\|A[\alpha \beta]^{T}+b\right\|^{2}$ with $A=$ $\left[{ }^{w} v_{1}{ }^{w} v_{2}\right]$ and $b={ }^{w} t_{c 1}-{ }^{w} t_{c 2}$, thus having a solution $\left[\alpha^{*} \beta^{*}\right]^{T}=-\left(A^{T} A\right)^{-1} A^{T} b$. The ball location can be estimated just using the scaling factor $\alpha$, but is more convenient to use also $\beta$ :

$$
{ }^{w} t=\left(\alpha^{* w} v_{1}+{ }^{w} t_{c 1}+\beta^{* w} v_{2}+{ }^{w} t_{c 2}\right) / 2 .
$$

Similarly, the ball radius could be obtained simply as $R=\alpha^{*}$, but once more is more convenient to average the scalings $R=\left(\alpha^{*}+\beta^{*}\right) / 2$.

\section{EXPERIMENTS}

\subsection{Experimental set-up}

The experimental set-up consists in a virtual world (the iCub simulator) observed by the robot's stereo cameras. The world is constituted by a textured background, a uniformly colored table and uniformly colored objects, including a ball which will be the subject of tracking. The ball trajectory is an helix with a diameter of $2 m$, starting $1.5 m$ away from the robot and moving with uniform velocity until $2.5 \mathrm{~m}$.

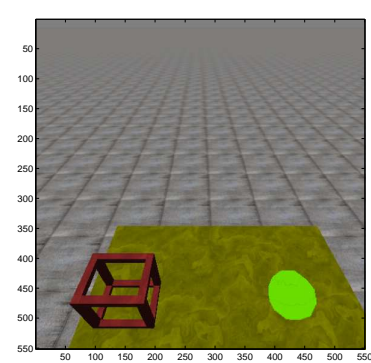

(a)

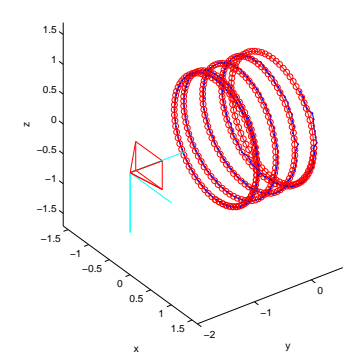

(b)
Figure 1: Left: first image of the sequence used for evaluation. Right: ball's motion follows an helicoidal trajectory moving away from the robot.
Fig. 1 shows both the simulator scenario and the 3D helicoidal imposed trajectory. The reason to use a simulated world in the evaluation of the algorithm is that it allows us to obtain the ground truth $3 \mathrm{D}$ position of the ball and thus compute the absolute tracking error achieved by different methods. Also, the intrinsic and extrinsic camera parameters are known which is useful to rule our errors thad could arise from camera calibration. We show the performance of our image segmentation method with the proposed Gaussian Mixture segmentation (Greggio et al., 2010a), (Greggio et al., 2010b), and the ellipse fitting algorithm (LCSE) (Greggio et al., 2010c). Then we evaluate our method to reconstruct the 3D position of the ball from the 2D ellipse parameters comparing monocular and binocular approaches.

\subsection{Image Segmentation by means of Gaussian Mixtures}

Fig. 2 shows the results of our image segmentation by means of Gaussian Mixtures algorithm applied to the images captured from the the iCub simulator. Here, we collected three sets of images: (1) is a scenario containing a large ball and a non-spherical object; (2) contains a small ball over the table; and (3) contains a small ball against the background. For each set, we show in Fig. (a) The original image, as captured by the camera; (b) The color segmented image, (c) the cost function as function of the number of mixture components, and (d) the behavior of the whole loglikelihood as function of the number of iterations.

Learning the right number of color components (i.e. mixture components) within a colored image is a difficult task. This is because a general colored image is supposed to contain a huge number of the three fundamental color combination, especially on modern devices. Therefore, the number of mixture components needed to represent the image at best rapidly rises up excessively, becoming too high, resulting in an excessive computational burden. Our approach is able to segment the images with a good accuracy (all the important features of the image are reproduced), while performing a lower computational burden against the other state-of-the-art techniques for a deeper comparison see (Greggio et al., 2010b). 
(1)

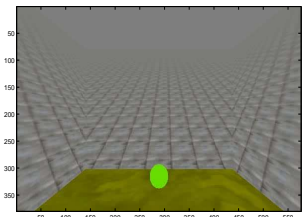

(a)

(2)

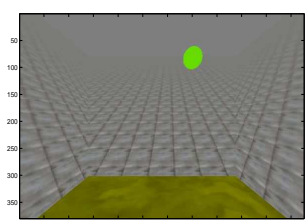

(a)

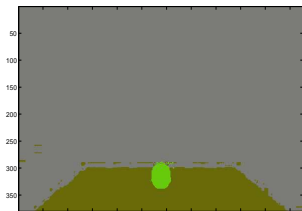

(b)

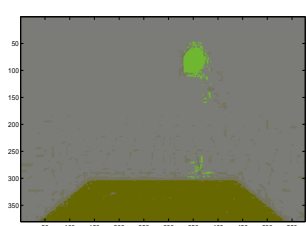

(b)

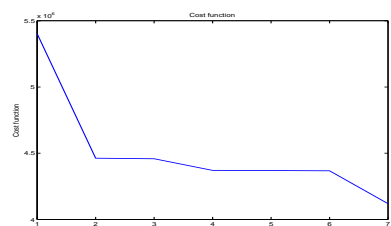

(c)

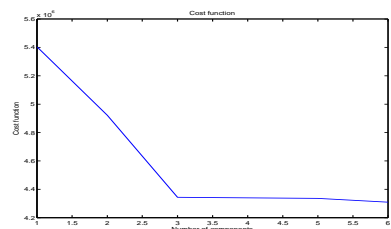

(c)

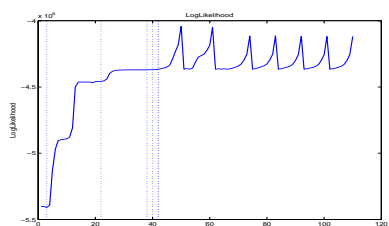

(d)

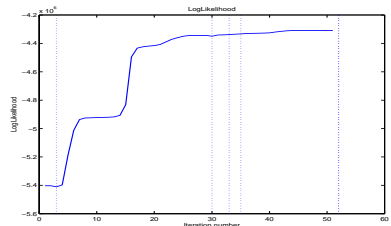

(d)

Figure 2: Image segmentation by means of Gaussian Mixtures. (a) The original images, as captured by the camera; (b) the color segmented images, (c) the cost function as function of the number of mixture components, (d) the cost function as function of the number of iterations, and (e) the behavior of the whole log-likelihood as function of the number of iterations.

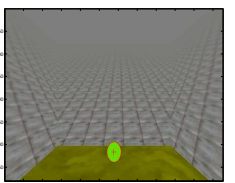

(a)

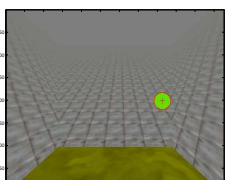

(b)

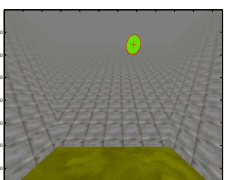

(c)

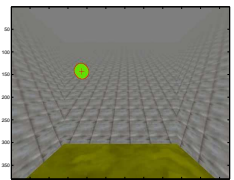

(d)

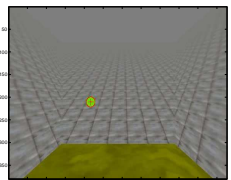

(e)

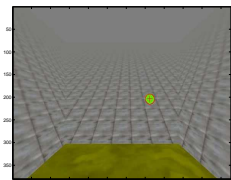

(f)

Figure 3: Ball reconstruction image sequence. Images at top row represent the original images with the superimposed ellipse.

\subsection{Ellipse Fitting}

Fig. 3 shows the results of ellipse fitting algorithm. Images at top row represent the original images with the superimposed ellipse. The ellipse fitting technique performs equally better when the ellipse is bigger or smaller. Of course, when the pattern's resolution decreases (e.g. when the target is small) the fitting precision may lack some accuracy, although this is merely a question of resolution rather than the algorithm itself.

\subsection{Ball Monocular 3D Localization}

In this section we present the results of ball 3D localization using a single camera. Fig. 4 shows the tracking errors along time and the $3 \mathrm{D}$ reconstructed trajectories. The generated trajectories are shown in red while the estimated trajectory is represented in blue. Due to the similarity among the analyzed ellipse fitting methods, in the following experiments we only use the LCSE ellipse fitting approach (Greggio et al., 2010c).

We can observe that the reconstructed trajectory follows closely the true one. The tracking errors grow slightly for increasing distances, which is a natural consequence of the decreasing size of the ellipse in the image and the consequent increase of discretization errors and estimation variance. Anyway, the absolute error is always kept below $7 \mathrm{~cm}$.

\subsection{Comparison Between Monocular and Binocular Reconstructions}

In this section we perform the reconstruction of the ball's position using a binocular approach. The used baseline is $10 \mathrm{~cm}$, matching approximately the structure of the iCub robot head. We consider two scenarios corresponding to cases where prior knowledge of the ball radius is absent or present: (i) when ball radius is unknown we use stereo triangulation, as described in section 2.3; (ii) when ball radius is known we use the mean of the two monocular reconstructions associated to each of the cameras of the binocular pair. Furthermore, for the binocular case, we consider the possibility of having uncertainty in the vergence angle formed by the cameras. This is a common case in robots with moving eyes and is due to either mechanical effects (backlash, miscalibration) or asynchronous acquisition of image and motor angles. Results are shown in Fig. 5. The top row shows the results of stereo triangulation with unknown ball 


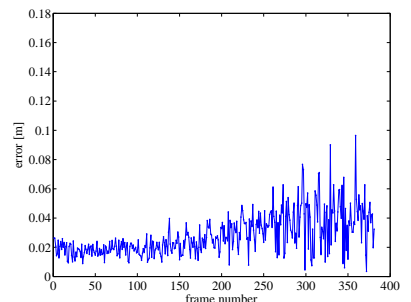

(a) Reconstruction error vs time. (b)

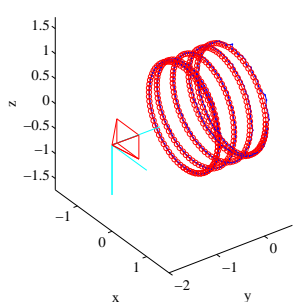

(b) $3 \mathrm{D}$ reconstructed trajectory of the ball.

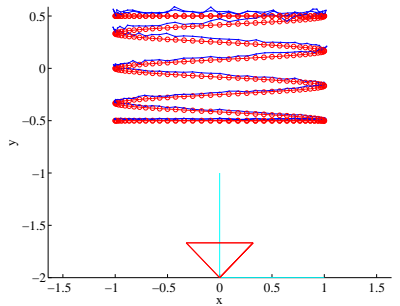

(c) 3D trajectory, top view.

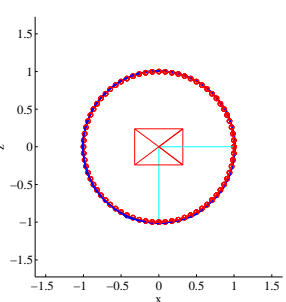

(d) 3D trajectory, behind camera frontal view.

Figure 4: 3D monocular reconstruction of the trajectory of the ball based on the LCSE ellipse fitting. Ground truth and reconstructed trajectories are in red (circles) and blue (dots). The average reconstruction error is $2.8 \mathrm{~cm}$ for reconstruction depths larger than $1 m$

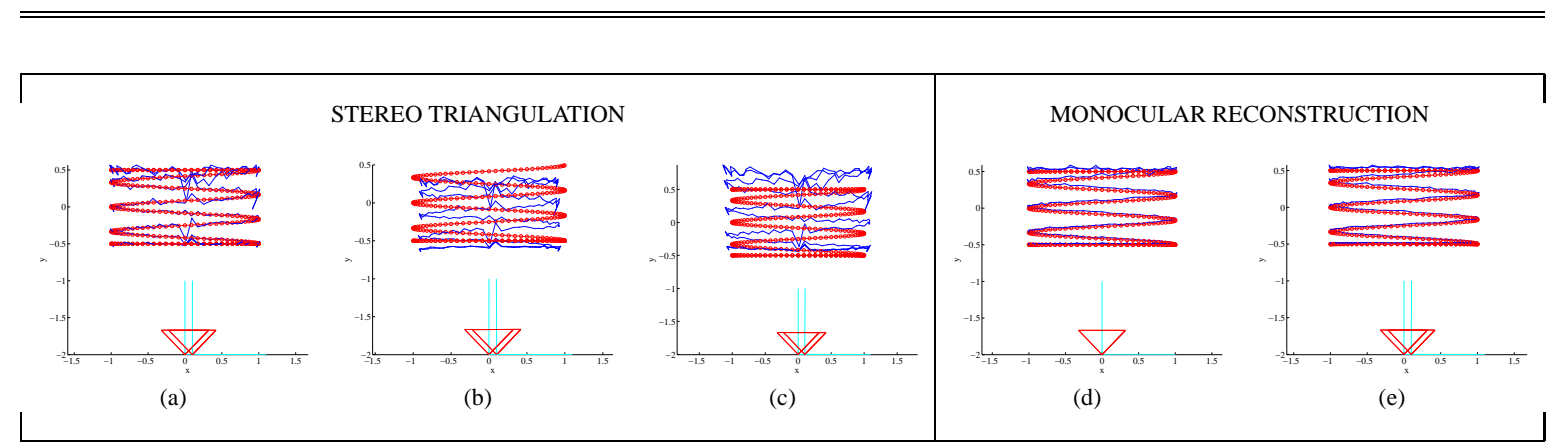

Figure 5: Comparing monocular and binocular reconstruction. The left set (a-b-c) shows stereo triangulation, when ball size is unknown. Stereo reconstruction with zero, $-0.2^{\circ}$ or $+0.2^{\circ}$ vergence errors $(\mathrm{a}, \mathrm{b}$ and $\mathrm{c}$ ). The bottom set (d-e) considers known ball size. Monocular reconstruction (d). Average of two monocular reconstructions, with vergence error of $+0.2^{\circ}(\mathrm{e})$.

radius. It is obvious a bigger variance of the estimate with respect to the monocular case, even in the absence of vergence angle errors (compare Fig. 5(a) and (d)). This shows the well known sensitivity of the stereo triangulation algorithm to small errors in the input data for small baselines. If the ball size is known, the binocular method copes much better with vergence angle error (Fig. 5(e) vs (b) and (c)). However, if the ball size is known, the monocular method shows roughly the same performance (Fig. 5(d) vs (e)).

\section{CONCLUSION}

This paper presents a real-time 3D ball tracking system including all processing stages, from image segmentation, 2D feature extraction and 3D reconstruction. All stages of the processing pipeline were developed taking both quality and computation time into account and are carefully described in the paper. The segmentation algorithm uses a clustering based ap- proach in joint color-space coordinates, using a novel greedy optimization of Gaussian Mixture parameters that overcomes related techniques in video segmentation. The 2D ellipse fitting method was designed for improved robustness to singular cases and is competitive with alternative methods. The $3 \mathrm{D}$ reconstruction method is simple, elegant and makes effective of all ellipse parameters obtained from the image. We performed experiments with a realistic simulator of the iCub robot. The full tracking method was evaluated by comparing tracking errors with ground truth values. Monocular and binocular approaches were tested, including the presence of errors in vergence angle measurements. This is a frequent case in robotic heads with moving vergence and the results obtained confirmed our empirical tests on the iCub robot: Vergence angle errors propagate significantly to $3 \mathrm{D}$ reconstruction errors in this robot. We showed that $a$ priori knowledge of the ball radius can reduce significantly the variance of the $3 \mathrm{D}$ estimates. Although it may not be possible in practice to obtain this knowledge in advance, our future work will focus on the 
online estimation of the ball size and the demonstration of its influence in the 3D reconstruction quality.

\section{ACKNOWLEDGEMENTS}

We thank Cecilia Laschi and Paolo Dario for their support. This work was supported by the European Commission, Project IST-004370 RobotCub and FP7-231640 Handle, and by the Portuguese Government - Fundação para a Ciência e Tecnologia (ISR/IST pluriannual funding) through the PIDDAC program funds and through the projects BIO-LOOK, PTDC / EEA-ACR / 71032 / 2006, and DCCAL, PTDC / EEA-CRO / 105413 / 2008.

\section{REFERENCES}

Andriluka, M., Roth, S., and Schiele, B. (2010). Monocular $3 \mathrm{~d}$ pose estimation and tracking by detection. IEEE Conference on Computer Vision and Pattern Recognition (CVPR 2010), San Francisco, USA.

Assfalg, J., Bertini, M., Colombo, C., Del Bimbo, A., and Nunziati, W. (2003). Semantic annotation of soccer videos: automatic highlights identifcation. Computer Vision and Image Understanding, 92:285-305.

Carpin, S., Lewis, M., Wang, J., Balakirsky, S., and Scrapper, C. (2006). Bridging the gap between simulation and reality in urban search and rescue". In Robocup 2006: Robot Soccer World Cup X.

Cross, G. and Zisserman, A. (1998). Quadric reconstruction from dual-space geometry. Int. Conf. on Comp. Vision $(I C C V)$, pages $25-31$.

Davis, J. W., Morison, A. W., and Woods, D. D. (2006). An adaptive focus-of-attention model for video surveillance and monitoring. Machine Vision and Applications, 18(1):41-64.

Fitzgibbon, A., Pilu, M., and Fisher, R. (1999). Direct least square fitting of ellipses. IEEE Trans. PAMI, 21:476480.

Forsyth, D. A. and Ponce, J. (2002). Computer Vision: A modern approach. Prentice Hall, NY.

Fossati, A., Dimitrijevic, M., Lepetit, V., and Fua, P. (June 17-22, 2007). Bridging the gap between detection and tracking for $3 \mathrm{~d}$ monocular video-based motion capture. IEEE Conference on Computer Vision and Pattern Recognition, Minneapolis, pages 1-8.

Gokturk, S., Bouguet, J., and Grzeszczuk, R. (2001). A data-driven model for monocular face tracking. ICCV01, pages 701-708.

Greggio, N., Bernardino, A., Laschi, C., Dario, P., and Santos-Victor, J. (2010a). Self-adaptive gaussian mixture models for real-time video segmentation and background subtraction. IEEE 10th International Conference on Intelligent Systems Design and Applications (ISDA), Cairo, Egypt.
Greggio, N., Bernardino, A., Laschi, C., Santos-Victor, J., and Dario, P. (2010b). An algorithm for the least square-fitting of ellipses. IEEE 22th International Conference on Tools with Artificial Intelligence (ICTAI 2010), Arras, France.

Greggio, N., Bernardino, A., Laschi, C., Santos-Victor, J., and Dario, P. (2011). Real-time 3d stereo tracking and localizing of spherical objects with the icub robotic platform. Journal of Intelligent \& Robotic Systems, pages 1-30. 10.1007/s10846-010-9527-3.

Greggio, N., Bernardino, A., and Santos-Victor, J. (2010c). Sequentially greedy unsupervised learning of gaussian mixture models by means of a binary tree structure. 11-th International Conference on Intelligent Autonomous Systems (IAS-11) 2010 - Aug 30, Sept 1.

Hartley, R. and Zisserman, A. (2000). Multiple view geometry in computer vision. Cambridge University Press.

Kwolek, B. (2004). Real-time head tracker using color, stereovision and ellipse fitting in a particle filter. $I N$ FORMATICA, 15(2):219-230.

Maini, E. S. (2006). Enhanced direct least square fitting of ellipses. IJPRAI, 20(6):939-954.

Menegatti, E., Silvestri, G., Pagello, E., Greggio, N., Mazzanti, F., Cisternino, A., Sorbello, R., and Chella, A. (2008). 3d realistic simulations of humanoid soccer robots. International Journal of Humanoid Robotics, 5(3):523-546.

Ottlik, A. and Nagel, H.-H. (2008). Initialization of modelbased vehicle tracking in video sequences of innercity intersections. International Journal of Computer Vision, 80(2):211-225.

S. Balakirsky, S. C., A.Kleiner, Lewis, M., Visser, A. Wang, J., and Ziparo, V. (2007). Towards heterogeneous robot teams for disaster mitigation: Results and performance metrics from robocup rescue. Journal of Field Robotics.

Shi, Y., Qian, W., Yan, W., and Li, J. (2007). Adaptive depth control for autonomous underwater vehicles based on feedforward neural networks. International Journal of Computer Science \& Applications, 4(3):107-118.

Stentz, A. (2001). Robotic technologies for outdoor industrial vehicles. Unmanned ground vehicle technology. Conference No. 3, Orlando FL, ETATS-UNIS (16/04/2001), 4364:192-199.

Tikhanoff, V., Fitzpatrick, P., Nori, F., Natale, L., Metta, G., and Cangelosi, A. (2008). The icub humanoid robot simulator. International Conference on Intelligent RObots and Systems IROS, Nice, France. 